\title{
Tweeting Traffic Image Reports on the Road
}

\author{
Daehan Kwak, Daeyoung Kim, Ruilin Liu, Liviu Iftode, Badri Nath \\ Department of Computer Science, \\ Rutgers University, Piscataway, NJ 08854-8091 \\ \{kwakno1, daeyoung.kim, rl475, iftode, badri\}@ cs.rutgers.edu
}

\begin{abstract}
As mobile phones have the ability to act as participatory sensors, we are beginning to witness the popularity of crowdsourcing and the sharing of traffic reports to improve the quality of the driving experience. This paper presents the architecture and implementation of a system called Social Vehicular Navigation (SVN), which allows users to generate and share geo-tagged image traffic reports called NaviTweets. Based on these traffic reports, Traffic Digests (concise snapshot summaries on the route of interest) are delivered to drivers to provide rich and reliable information supporting the route choice. These digests will complement factors such as the estimated travel time and assist the driver on their route choice decision making. The paper presents the initial design, along with a prototype implementation running on the Android platform, and details a user study conducted to evaluate the influence of providing traffic images on route-choice behavior.
\end{abstract}

Keywords-social networks; vehicular networks; navigation systems/applications; route choice; traffic images

\section{INTRODUCTION}

With the ever-expanding affordability of cars throughout the world, traffic congestion is a severe problem that can have a negative impact on the economy, the environment, and human sentiment. There are a myriad ongoing attempts to alleviate traffic congestion, for example the infrastructure-based Intelligent Transportation Systems (ITS) such as on-ramp flow meters, traffic cameras, number plate recognition systems, and electronic informational displays (e.g. Variable Message Sign) along the roadways. Today, the most widely used solutions are infrastructure-less, named so because they use floating car data to determine traffic speed and to identify traffic congestion. Provided to the onboard navigation systems, this information can be used to calculate the fastest route. The infrastructure-less (or crowdsourcing) approach can be classified into two types: push and pull. The majority of today's systems anonymously pulls GPS-based speed and location information from the client's mobile or navigation system to provide real-time traffic information. The push type is based on user participation, where they push traffic reports in a richer context (e.g. the location of red light cameras and speed traps, the degree of traffic congestion, images, etc.) onto the server to share with other users. Waze [1] is an example of a navigation app that anonymously pulls speed and location information while providing an interface for drivers to push traffic reports.

Traditionally, traffic reporting has been done primarily by the police, state departments of transportation, drivers reporting

This work was supported in part by the National Science Foundation (NSF) grant CNS-1111811. by phone, and also by traffic reporting companies. Such information is aggregated and then either resold or redistributed directly to the public, broadcast on-air by radio and TV stations, or used as traffic data for in-car navigation systems. Live traffic status reports are becoming more common and easily accessible, with traffic congestion maps available via online maps, mobile phones, and GPS devices. Nowadays, participation by the public in providing traffic reports is becoming popular, because it is easy for users to report and share traffic information with one another via smartphones.

Traffic information can influence drivers' route choice behavior, and consequently, guide them to less congested routes. When planning a route, current technologies collect and use realtime traffic information to calculate the route and then present a recommended list of alternative routes (normally two or three options) based on factors such as the Estimated Time of Arrival (ETA) or the shortest distance. Based on the list of optional routes, it is up to the driver to decide which route to take. Because ETA is the main factor that can be used in route decision, the design of vehicle navigation systems does not take into account other semantically richer information to support decision making and satisfaction of route selection.

This paper focuses on how to provide a secondary level of detail using crowdsourced traffic images to support drivers in the selection of routes. To do so, we propose a system called Social Vehicular Navigation (SVN), which allows users to share image traffic reports, called NaviTweets. The users' shared traffic reports are geo-tagged onto a map, called Social Traffic Map (a map representation). Based on many NaviTweets, a Traffic Digest is used to summarize the information of the route that is of interest to the user. Once the Traffic Digest is received, the information is displayed in a user-friendly way to the drivers to assist them with route selection. We explain the functions of the proposed SVN model in abstraction layers. Also, the system design for the SVN prototype implemented on the Android platform is presented along with results from a questionnaire survey to evaluate the usage of traffic images in route choice.

This paper is organized as follows. The next section provides preliminaries by presenting related work. The proposed model for SVN is discussed in section III. The SVN system design and its prototype implementation is presented section IV and V, respectively. In section VI, the results of a user study based on a questionnaire survey is presented. Section VII presents a discussion and suggested future work and finally the conclusion of this paper in section VIII. 


\section{RELATED WORK}

\section{A. Participatory Crowd Sensing}

Crowdsourcing of traffic information is becoming popular because smartphones are easily used to act as participatory sensors. Waze [1] is a navigation app that differs from traditional navigation systems in that crowdsourcing via users provides traffic reports to a central server, where such information is used to provide real-time routing and traffic reports. Here, push buttons are used to share road reports like the degree of traffic, police speed traps or accidents. More recently, apps such as Inrix Traffic [2] have started to incorporate user-based crowdsourced traffic reports, and after Google's recent acquisition of Waze, it started to add social traffic reports into its mapping business. Trapster [3] is also a community-based crowdsourcing app that improves the commute when users report and share the location of speed traps, red light cameras and speed radar, etc. In addition, recent research $[4,5,6,7]$ also talks about the potential from users sharing information regarding traffic, and we are starting to witness widespread use of these techniques.

\section{B. Vehicular Social Network}

Smaldone et al. [5] presented a framework called Vehicular Social Networks (VSN), which is an integration of social and vehicular networks, where the goal is to construct a periodic virtual social community for commuters who are traveling on the same roadways at the same time. As an example of VSN, the authors presented RoadSpeak, a voice chatting system over VSN, which is used to facilitate communications between commuters or a group of commuters to share common interests.

Another application of VSN shares driving experiences integrated into vehicle navigation, also called NaviTweet [6]. Drivers can create traffic-related "voice tweets" about the road, which are then aggregated into tweet digests and shared with other drivers. With this information, drivers can instruct the navigator to avoid or choose certain routes. The NaviTweet version described in this paper differs in several ways. First, images are used instead of the voice and a questionnaire survey was conducted to study the influence of traffic images on drivers' route choices. Second, the digest algorithm differs in that the previous version was based on time and space, whereas the current version is based on time, space and causal order (for example, accident causes traffic congestion). Third, the current version of NaviTweet was implemented on the Google Maps [20] platform, whereas the previous version was implemented on OpenStreetMap (OSM) [21]. Finally, a more detailed version of the Social Vehicle Navigation model is described.

\section{Route Choice}

In the transportation field, route choice behavior is concerned with the decision-making process of route selection, and research has been conducted to understand this complex behavior $[8,9]$. Many factors influence the decision process. For example, there are observable attributes, such as travel time, cost, distance, fewest turns, trip purpose, and traffic information availability; and there are unobservable characteristics, such as age, gender, income, attitude, perception, personality, spatial abilities, and road network familiarity.

In previous studies, traffic images were not considered a criterion of the route decision, thus to the best of our knowledge, there is limited work on the usage of images in route choice behavior. However, due to the development of ITS, there are a few patent applications $[11,12]$ and some literature $[13,14]$ that apply or identify the usage of traffic cameras in route planning. Hanchett [11] proposed a method to install a series of image sensors spaced along major roads to provide images of traffic, which are sent to a main station and then distributed to users. Users have a receiver that displays the images so they can preview the route ahead and make route choices. A patent application by Adem et al. [12] proposed a navigation device that displays a route on a road map along with locations where visual traffic information exists. Visual traffic information comes from fixed traffic cameras, and viewing the video feed or still images allows the driver to assess the traffic conditions. The Highways Agency in the United Kingdom is making images from traffic cameras available to licensed organizations to provide traffic information to the public to enable better route planning [14]. Speirs et al. [13] described a research survey to evaluate the influence of providing public access to traffic camera images. Their results showed that combined with other sources of traffic information (e.g. speed/delay information or radio traffic news), traffic images provided an additional secondary level of detail and support drivers in making better decisions on their route choice. Up-to-date traffic camera images are also available to the public in the United States and can be accessed from the corresponding state's 511 website [15].

\section{Social VEHICle NAVIGATION MOdEL}

This section illustrates the functions of the proposed SVN model in abstraction layers, as shown in Figure 1. The functionalities of the SVN model are partitioned in a way similar to the Open Systems Interconnection (OSI) networking model, where each layer has independent functionalities and serves the layer above it.

Traffic Layer. The traffic layer is where the routing algorithm is executed to find the list of alternative routes based on criteria such as the shortest distance or the fastest route. It also stores and updates real-time speed or delay data on available road segments, where they are used to calculate ETA on each alternative route of interest. Information regarding route plans and traffic speed/delays for route segments flows up to the upper layer.

Social Traffic Layer. The social traffic layer consists of social traffic data, i.e. traffic reports in media files, such as images, that are geo-tagged on the road network map. Such traffic reports are crowdsourced from participants who are willing to share traffic information. Once the traffic layer calculates and recommends a list of routes, all the corresponding traffic reports that coincide with the routes are selected and passed to the presentation layer. The reason for this is to filter out traffic conditions that are reported, irrespective of the route that the driver is interested in.

Presentation Layer. When selecting a route, the user is typically interested in the information that is along the routes that are of interest. Drivers who receive too much information may become oversaturated and may have difficulty processing the information $[9,10]$. It would be redundant to view similar traffic reports on the route, so the Traffic Digest is used to summarize the set of NaviTweets based on the geo-tagged locations. 

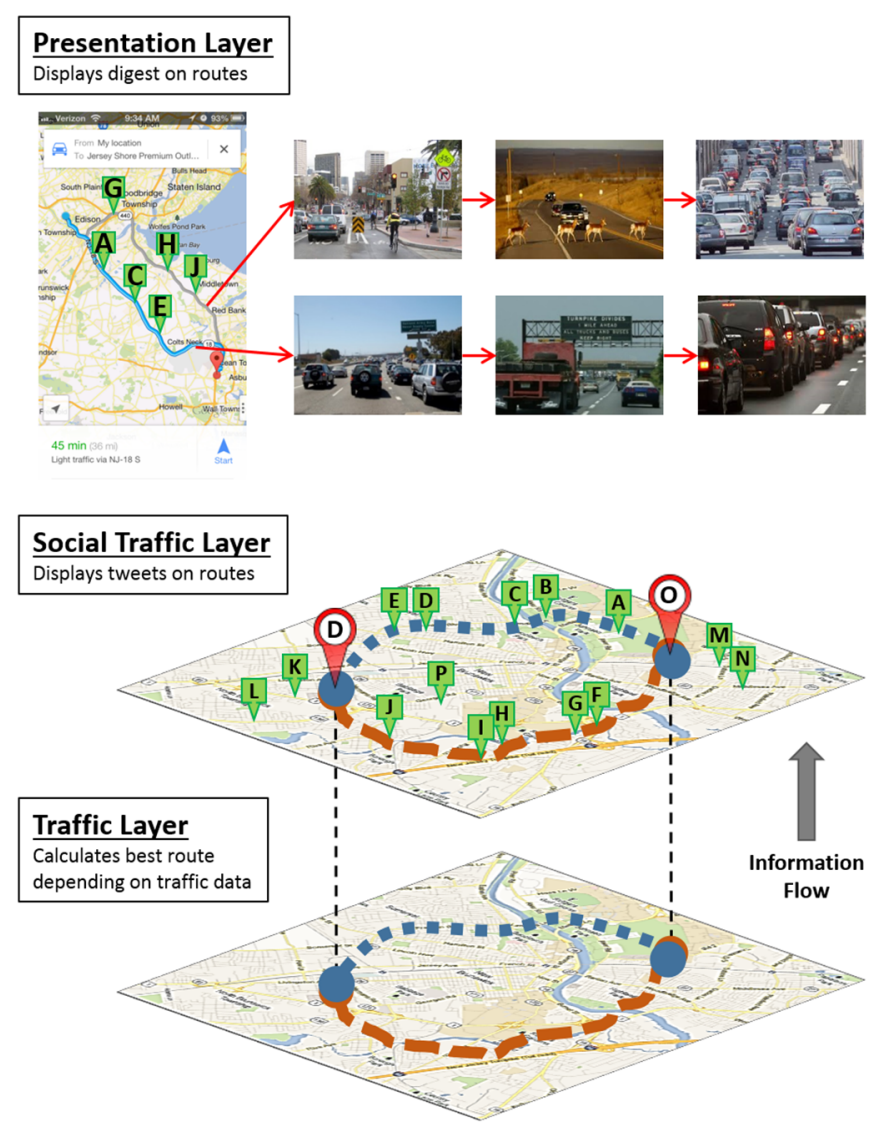

Fig. 1. Social Vehicular Navigation Model.

For example, one of the routes consists of a set of NaviTweets $\{A, B, C, D, E\}$ as shown in the middle of Figure 1. After the digest, a summary of the set, $\{A, C, E\}$ is displayed. Details on how the Traffic Digest works are presented in Section IV. Also, in the mobile version, users do not have the luxury of clicking on each geo-tag to view what the tweet is about. Thus, to reduce the cognitive load, it is important to provide a userfriendly interface so the user can sequentially view the NaviTweets as shown in the upper portion of Figure 1.

\section{System AND Design}

\section{A. Design Consideration}

A few key points that are adopted in the SVN design are listed below. The problem domain includes unique features, such as small time-to-live (TTL) events and low cognitive load, etc., which provide challenges as well as advantages. Key design considerations are explained below.

Computation and Communication. Limitations in computing power and energy on mobile devices make it important to offload computation (i.e. NaviTweet processing) and communications to a server. The client's computing processes and communications with the server should be kept to minimum.

$T T L$. A typical traffic event has a time-to-live (TTL) that lasts anywhere from several minutes to several days. For example, traffic jams rarely last more than a couple of hours, but construction can last for several days or weeks. A shorter TTL reduces the requirement for data durability. A TTL feature based on the traffic event is incorporated, such that NaviTweets can be safely deleted from the storage/cache after the TTL is up.

NaviTweets. NaviTweets are composed of photos or small videos. The size of such tweets is in the order of kilobytes or, at most, megabytes. The existence of many popular online services, e.g. Instagram [22], has shown that uploading and downloading photos through mobile devices can be handled by contemporary network architectures. On the other hand, lengthy video uploads can be challenging to accommodate. Photos or videos are taken as raw byte streams on mobile devices, and contain little cues for inferring content. User tagging or object detection using recognition techniques can be used to index the data more effectively.

Low Cognitive Load. SVN should minimize the driver's cognitive load when interacting with the application and comprehending the data. Drivers are accustomed to voice communication, so voice command or gestures are preferable whenever possible.

Scalability. SVN is designed as a scalable online service for all commuters to share traffic information. The architecture should accommodate a large number of users uploading or downloading data at the same time. A distributed architecture, or for example, cloudlets [18], can provide a natural solution to provide scalability. Moreover, a distributed architecture is favored due to the fact that most commutes occur between home and work within a reasonably stable and small geographic region. A local server cluster or cloudlet can handle the majority of requests in its locality.

Availability vs. Consistency. Drivers normally prefer to have control of up-to-date traffic information. If a distributed storage architecture is used, it is preferable to provide drivers with timely and inconsistent traffic information rather than outdated and consistent data. Moreover, community-based information can be inaccurate, e.g. false traffic alerts, so it is not necessary to emphasize consistency among the distributed servers.

Network Bandwidth. As network bandwidth varies according to traffic volume, it is expected that the aggregated network data traffic for uploading and downloading NaviTweets should vary in accordance with commuting traffic volume. This is assumed, based on the obvious fact that traffic events occur more oftenand more users are concerned-during peak rush hour.

\section{B. Client Design}

\section{1) Posting NaviTweets}

When posting a NaviTweet, it is important to catch as much information as possible, yet at the same time, minimize the cognitive load on the user. We propose two models for posting: active and passive. To minimize the cognitive load, the whole procedure should be completed in three commands, where each command is executed by either voice or gesture. The active model is for users who are actively willing to post NaviTweets. Figure 2 illustrates the flow of the active model.

When the variable $f$ is above a predefined threshold, the client device automatically detects a potential traffic event and takes a picture. This variable can be set by using factors such as the current speed or deceleration time interval, etc. Then, the 
user is prompted on whether to post the image or not. If the user agrees to post the NaviTweet, the user is prompted again on whether to annotate it. If agreed, a list of recommended tags is presented to the user to select via voice command. The passive model is designed for users to post NaviTweets whenever they choose to. The only difference from the active model is that whenever the user wants to post a NaviTweet, the user can either push a button or wave a hand over the proximity sensor, which triggers the camera. Once the picture is taken, the process is the same as above.

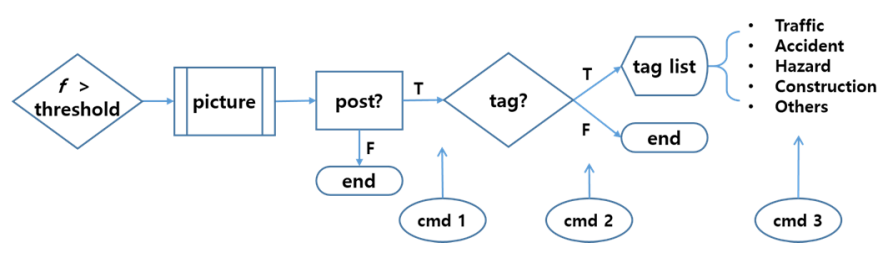

Fig. 2. Active Posting Model.

The optimal placement of the smartphone to take suitable pictures is one of several positions above the dashboard within the car. The general placement of the smartphone device in order to take images and provide a good view should be on a smartphone holder anywhere in the middle area above the dashboard or in the front windshield.

\section{2) Requesting Digest}

Once the traffic map layer performs the route calculation, a list of recommended routes is provided based on the routing algorithm. Users will request the NaviTweet digest on the routes that are of interest. The client device will send the road segments to the server and the user will be able to view the digest in a sequential series of events along the route to the destination.

\section{Server Design}

\section{1) Receiving NaviTweets}

When a tweet arrives at the server, the server updates a digest on the corresponding road segment based on the location of the tweet. Since each NaviTweet post contains a user-provided or context-inferred tag, the server can index and aggregate the photos based on the tags. We define five tags that can be associated with a photo: traffic, accident, hazard, construction, and others. Moreover, a causal order is defined between photos using the tags. A causal order is a happen-before relationship between events $\mathrm{X}$ and $\mathrm{Y}$. It indicates whether event $\mathrm{Y}$ is caused or influenced by an earlier event, $X$. Therefore, it is a semantic causal order. It is obvious that drivers prefer to know the reason for a traffic jam; for example, a lane closed for the rest of the day provides a completely different logical inference of future traffic than a malfunctioning car. So, causal order can also indicate which photo is more valuable to drivers. We assume the following simple causal order between traffic events, defined as $\mathrm{X} \rightarrow \mathrm{Y}$ ("X is a cause of $\mathrm{Y}$ "):

- Accident $\rightarrow$ Traffic

- $\quad$ Construction $\rightarrow$ Traffic

- $\quad$ Hazard $\rightarrow$ Traffic

\section{2) Traffic Digests}

When a user requests a digest, the server will handle the request and respond with a series of NaviTweet results, each of which is a well-formed data structure, containing the location, time, and media content. The Traffic Digest consists of three processes: selection, digestion, and composition. Each step is examined in detail below.

Selection. First, all NaviTweets posted on the requested road segment within the last TTL minutes are called. This operation is a simple iterative call on the storage system, with each road segment and current time as the parameters.

Digestion. The server then summarizes the potentially large return set from the tweet selections into a human-readable set of tweets. The resulting set is the Traffic Digest. The motivation for digestion is twofold. First, it is not feasible to send a driver all the relevant tweets, because this will take up too much time browsing the photos. Secondly, digestion can be used to eliminate redundancy in the tweets in order to give drivers a concise and up-to-date view of the traffic.

The digestion algorithm first assigns each tweet to its road segment. Assume the length of each road segment has an upper bound. Those road segments that exceed the bound are divided into shorter ones. The algorithm then iterates through each road segment, and extracts and returns the latest tweet in each tag category. Moreover, a tweet with the tag "traffic jam" will only be included in the resulting set when there is no tweet tagged accident, construction or hazard. This rule is used to enforce causal order between tweets. In other words, if tweet $\mathrm{X}$ represents a traffic accident, and tweet $Y$ represents a traffic jam on the same road segment, then tweet $X$ is returned while tweet $\mathrm{Y}$ is not, the reason being that, "accident" is more informative than the consequence (i.e. traffic jam). Digestion is performed and subsequently produces a concise final set of tweets for the composition stage.

Composition. The tweet digest produced is not ordered in either location or time. It is the role of Composition to sort the tweets in increasing distance order and annotate each photo with location and time. This provides a natural view for drivers as to reasons for the traffic ahead. The resulting ordered list of NaviTweets is then sent to the client.

\section{PROTOTYPE}

\section{A. Implementation}

The prototype SVN system is based on many of the available online services. The SVN client runs on the Andriod 4.1+ mobile platform and uses the Google Places API, the Google Directions API and the Google Maps API to compute and show the alternative routes, given the origin and destination. Because it is important to offload computation, the digest is fetched from the NaviTweet engine server using the developed NaviTweet API.

The NaviTweet engine server is currently set up on Ubuntu 12.04 LTS, and the server was configured with Apache 2.2, PHP 5.3 to process web requests from the client application. In addition, MySQL 5.5 was deployed on the server to store the tweets as well as metadata, such as the timestamp, location, and labeled categories. The Google Directions API was used to obtain the routes between origin and destination. A set of 
NaviTweet APIs provides functionalities for the client. The PostTweet API and the GetDigest API work as mentioned in Section IV-C.1) and IV.C.2). In addition, the GetTweet API was implemented to support the client in fetching NaviTweets using image_id.

\section{B. NaviTweet Scenario}

In this section, a usage scenario for the SVN system is presented to illustrate the workflow (shown in Figure 3) and the user interface (shown in Figure 4) of the SVN system.

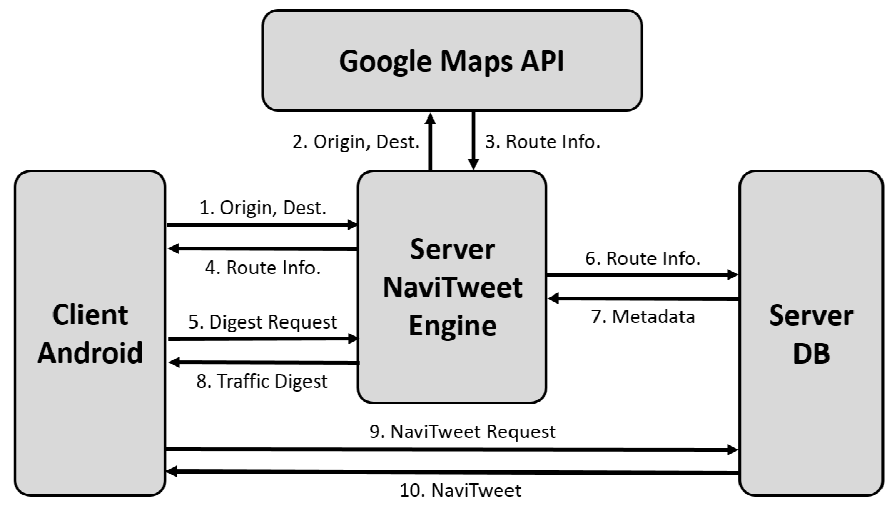

Fig. 3. SVN System Design.

When the user first launches the client application, the origin and destination input screen is shown, as seen in Figure 4(a). After introducing the origin and destination, location information for both is transmitted to the NaviTweet engine. The NaviTweet engine passes the origin and destination to the Google Maps API and receives the calculated route information. Route information is then returned to the client, and thus, the user can see the alternative route list and the corresponding ETAs as shown in Figure 4(b).

Upon selecting a route, users may want to check the traffic events happening along the route. In this case, the user presses the "View" button behind any alternative, and the request will be passed to the NaviTweet engine. Based on the route information and the metadata of tweets, the NaviTweet engine computes the digest and returns it to the client, which triggers the application to jump to the screen shown in Figure 4(c). The events are geo-located in the map, and the image of the first event is shown on the bottom half of the screen. The user can slide the screen to the left or right to navigate through images one by one, and such action will trigger the client to fetch the next image from the server DB. The location tag changes from green to cyan as the corresponding image is viewed. Finally, after deciding which route to take, the user presses the "Go" button and then starts navigation, as shown in Figure 4(d). While driving, if wishing to post a NaviTweet, the user can either press the button labeled "Tweet" located on the upper left corner of Figure 4(d) or wave a hand over the proximity sensor.

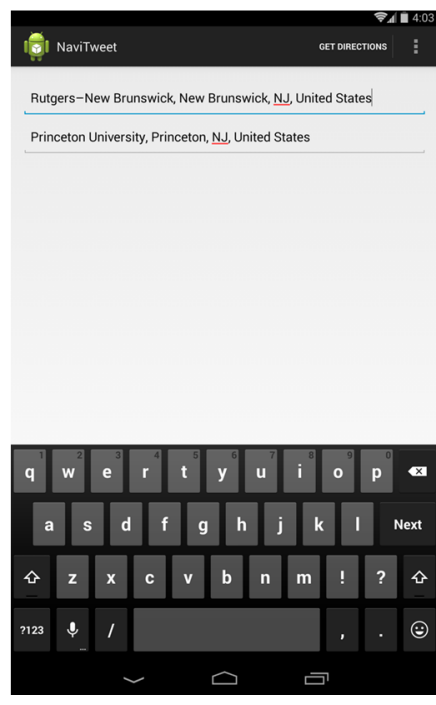

(a) Origin and destination input
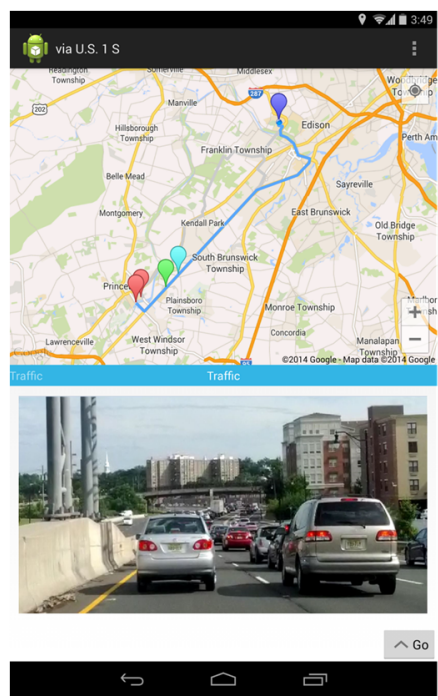

(c) Traffic view
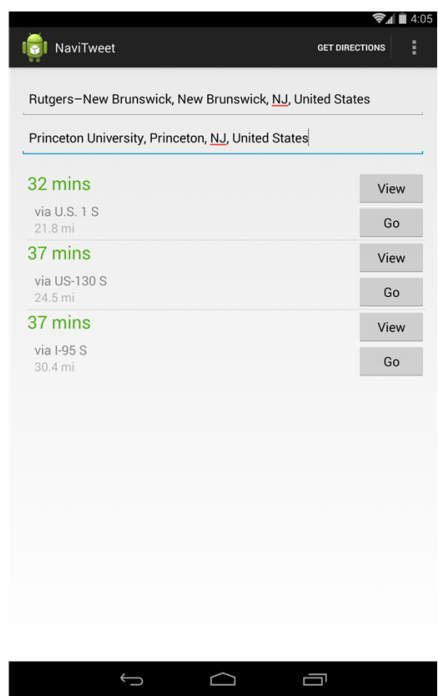

(b) Route view and selection

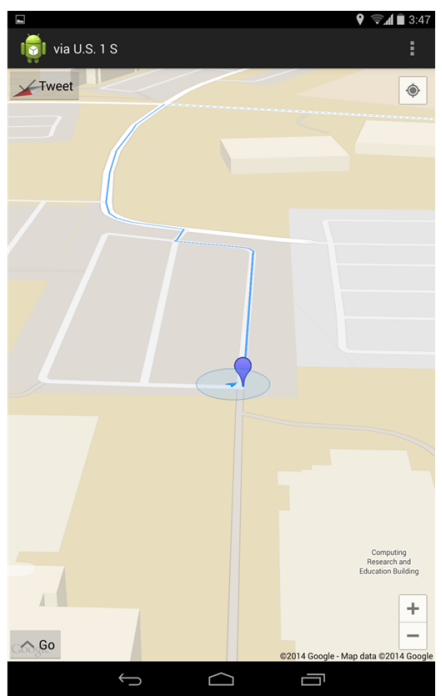

(d) Navigation and tweet
Fig. 4. Client Prototype.

\section{USER STUDY}

The motivation for implementing SVN was to develop a crowdsourced application and provide additional detailed information using images to support the driver as to route choice. A user study was designed to answer the following main objectives:

- factors that influence drivers when making initial decisions on route choice

- whether the use of traffic images influences drivers in route choice

- occasions that prompt willingness to use traffic images in route choice 
TABLE I. SURVEY QUESTIONNAIRE OUTLINE

\begin{tabular}{ll}
\hline \multicolumn{1}{c}{ Section } & \multicolumn{1}{c}{ Questions } \\
\hline \hline Participant Profile & Q1. Gender \\
& Q2. Age range \\
& Q3. Years of driving \\
\hline Traffic Information Source & Q4. Traffic information source preference \\
& Q5. Route choice preference \\
& Q6. Navigation system preference \\
& Q7. Criteria considered in route choice \\
& Q8. Occasions for the use of navigation \\
& Q9. Accuracy of ETA \\
& Q10. Other effective criteria in route choice \\
\hline Route Choice Behavior (w/o image) & Q11. Route selection \\
& Q12. Rate criteria based on decision in \#11 \\
Route Choice Behavior (w/ image) & Q13. Route selection \\
& Q14. Rate criteria based on decision in \#13 \\
\hline Influence of Traffic Images & Q15. Rate how image would support route choice \\
& Q16. Uses of traffic images \\
& Q17. Influence of traffic images \\
\hline
\end{tabular}

\section{A. Questionnaire Design and Outline}

An online web-based questionnaire using Qualtrics software [19] was developed with guidelines from previous survey questionnaires. Questions were either selected and modified to fit the study or inferred from results in previous literature based on 1) a study of the decision-making process of drivers considering alternative routes [16], 2) a study on the impact of traffic image camera usage on route choice [13], and 3) a study defining the criteria used in route selection [17].

The survey was divided into four sections: participant profile, preference as to source of traffic information, route choice behavior (with and without traffic images), and finally, the influence of traffic images. Table I illustrates the outline of the survey sections and the questions.

The survey questionnaire consisted of 17 questions, where five had additional sub-questions. Questions 1 through 3 collected basic profile information about the participant: gender, age range and years of driving. Questions 4 through 10 asked the participants about their preferences as to the various types of traffic information sources or media, what they rely on when choosing a route (e.g. experience or navigation system), type of navigation system they use, what criteria they currently use when selecting a route (e.g. distance, ETA, name of road or highway, turn-by-turn directions), in what instance they consider using a navigation system, how accurate they thought ETA was, and what other secondary criteria they thought was useful to support them in route selection.

For questions 11 and 12, the participants were shown a map with two routes. The source of the map image was an actual screen shot from the mobile version of Google Maps on a weekday at the $5 \mathrm{pm}$ rush hour, where the origin was the university campus and the destination was a grocery store. Both routes had the same ETA ( 25 mins), where route 1 was on a highway with a distance of 7.2 miles and route 2 was on a local road at 7.8 miles. Participants were then asked to choose the route they would travel, assuming that the area was unfamiliar. Then, they were asked to rate the criteria they used to make their decision. The criteria Google Maps offered was road or highway name, distance, estimated travel time (ETA), and color-coding roads (green, yellow, red) to indicate the severity of traffic congestion. Questions 13 and 14 asked the same questions as questions 11 and 12, respectively, but images were added to the route. The images were real images taken by the app, where both routes were driven simultaneously by two vehicles at the time when the screen shots were taken, as mentioned above. Participants were then asked to select a route with the additional traffic image information and rate the criteria they used to make their decision. Finally, for questions 15 through 17, the participants were asked about the usefulness of the traffic images and, if the participant was provided with such an app, on what occasions they would use the traffic images, and what influence traffic images would have on their trip.

To measure the participant's preferences, multiple choice questions with single/multiple answers and a Likert-type scale of five points (Strongly Don't Consider; Don't Consider; Neutral; Consider; Strongly Consider) were used for the questions. No information on the NaviTweet app (usage of traffic images) was mentioned to participants until Question 13. The time to complete the survey was estimated at about 20 mins.

TABLE II. PARTICIPANT PROFILE $(N=73)$

\begin{tabular}{lll}
\hline Question & Response & Count $(\%)$ \\
\hline \hline Gender & Male & $42(58 \%)$ \\
& Female & $31(42 \%)$ \\
\hline Age Range & $18-30$ years & $56(77 \%)$ \\
& $31-40$ years & $13(18 \%)$ \\
& $41-50$ years & $3(4 \%)$ \\
& $51-60$ years & $1(1 \%)$ \\
\hline Years of driving & Less than 1 year & $10(14 \%)$ \\
& $1-3$ years & $23(32 \%)$ \\
& $4-5$ years & $15(21 \%)$ \\
& $5-10$ years & $17(23 \%)$ \\
& More than 10 years & $8(11 \%)$ \\
\hline
\end{tabular}




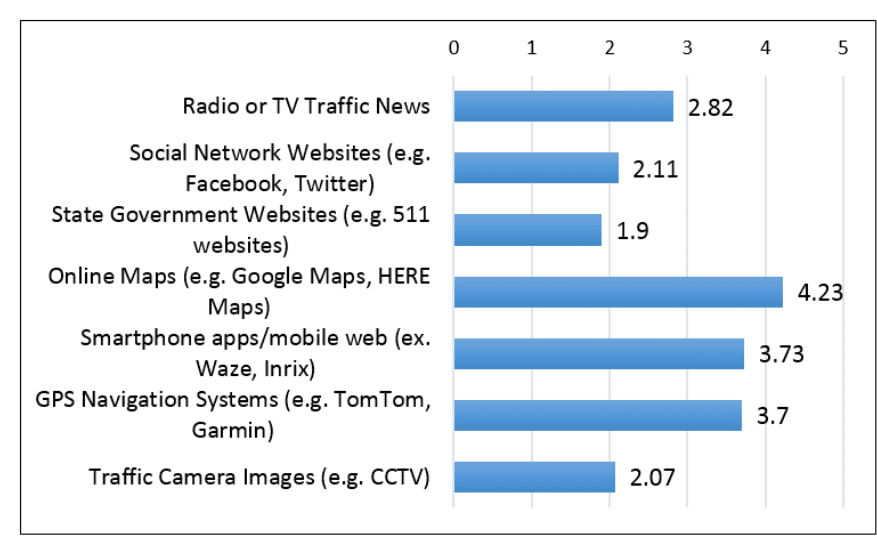

(a) Q4. Source of traffic information preference.

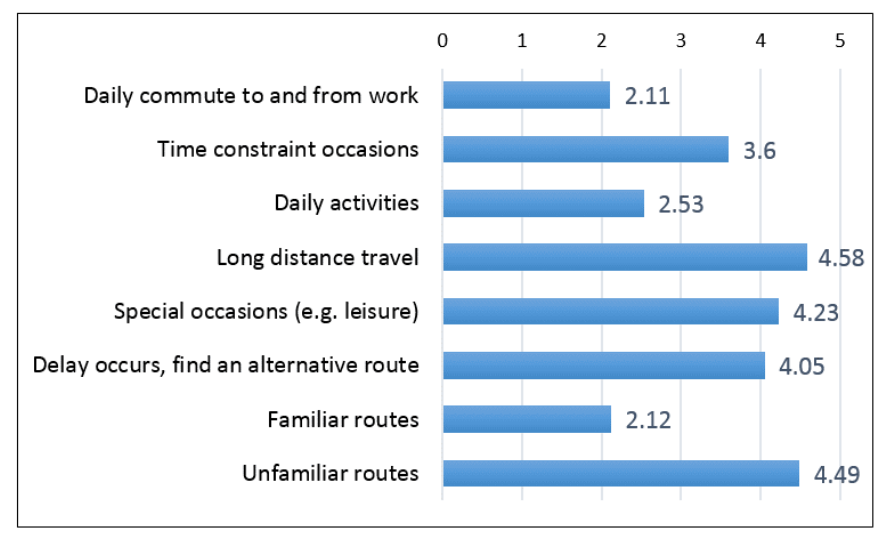

(c) Q8. Occasions in the use of navigation.

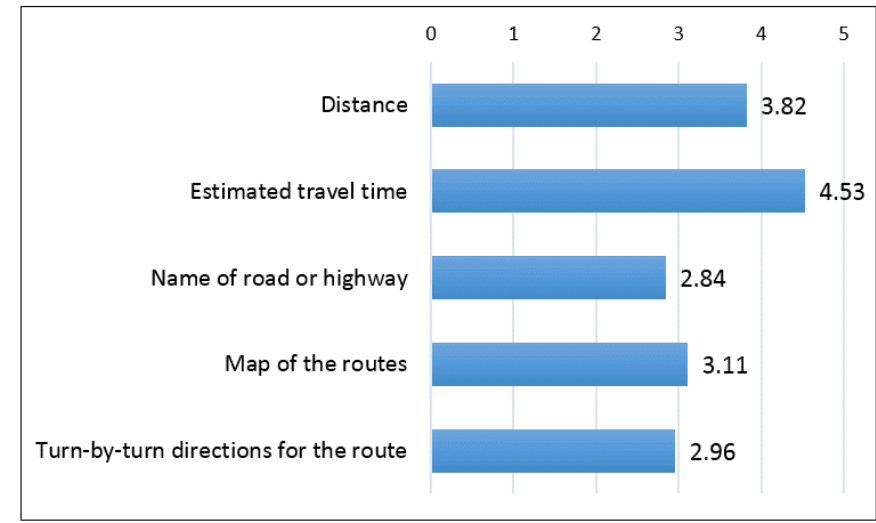

(b) Q7. Criteria considered in route choice.

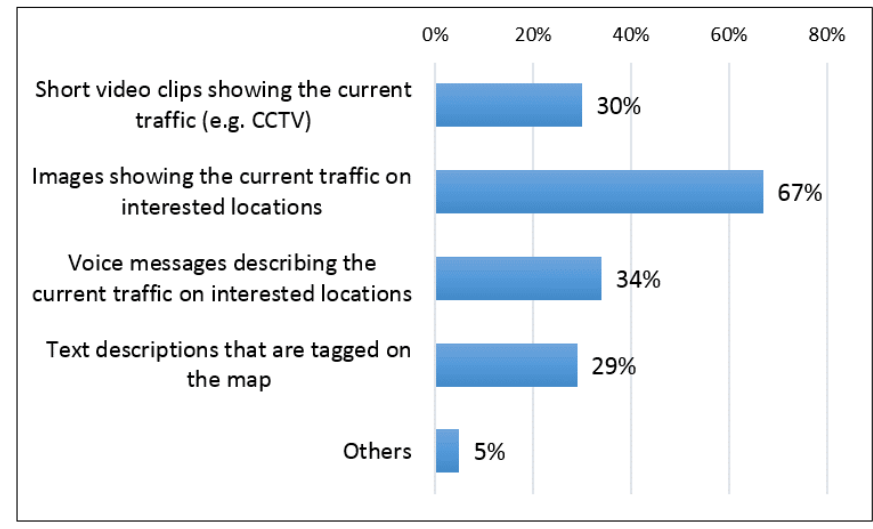

(d) Q10. Other effective criteria in route choice. (Multiple answer question)

Fig. 5. Survey Questionnaire Results (Q4, Q7, Q8, Q10)

\section{B. User Study Results}

Participant Profile. The web-based questionnaire was distributed to university students and colleagues, and was advertised on social networks. The user study involved a total of 98 respondents, where 73 completed the survey and 25 did not. Survey data from respondents who did not finish the survey were excluded. All respondents resided in the United States and had a driver's license. Table II summarizes the results for participant profiles based on questions 1 through 3 .

Traffic Information Source. Participants were asked to indicate the information sources used when they searched for travel information (Q4). Figure 5(a) compares the average rankings of all seven types of information source. Overall, the traffic sources ranked as most considerable were online maps, smartphone apps, and GPS navigation. Also, the participants were asked what they mostly relied on when choosing a route in terms of percentages (Q5). Approximately $47 \%$ were based on past experience, and $52 \%$ on recommendations from a navigation system. Only $1 \%$ of respondents based reliance on the "others" category (where text entry was allowed), and notable written replies were "directions given by parents/friends/others" or "suggestions from others." The responses for Q6 on the type of navigation the participant used were $55 \%$ in-car navigation systems, such as TomTom or Garmin, and it was notable to see that $90 \%$ used smartphone navigation apps, such as Google Maps, Map Quest, Waze, etc. In Q7, the participants were asked to rate the criteria when making a route choice. Estimated travel time (ETA) was ranked the highest (4.53) followed by distance (3.82), as shown in Figure 5(b). Q9 asked how likely the participants considered using a navigation system for eight possible instances, and longdistance trips and unfamiliar routes were the top two rankings, as shown in Figure 5(c). This study looked at the impact of traffic images and how they can be used to confirm the traffic conditions when there is doubt as to the reliability of ETA, and it ranks what participants thought about the accuracy of ETA. A Likert-type scale of seven points was used for this question, and the results shown are $5 \%$, very accurate; $27 \%$, accurate; and $33 \%$, somewhat accurate. Details are shown in Figure 7(a). Before being provided with any information on the application, the participants were asked what criteria other than the traditional (e.g. ETA, distance) they thought was useful in decisions about route choice (Q10). About 67\% answered that traffic images showing the current traffic helped when selecting a route, as seen in Figure 5(d). 


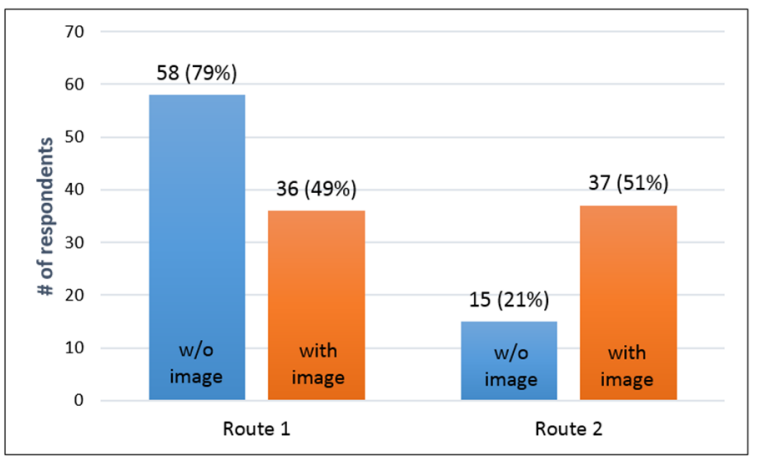

(a) Q11, Q13. Route selection.

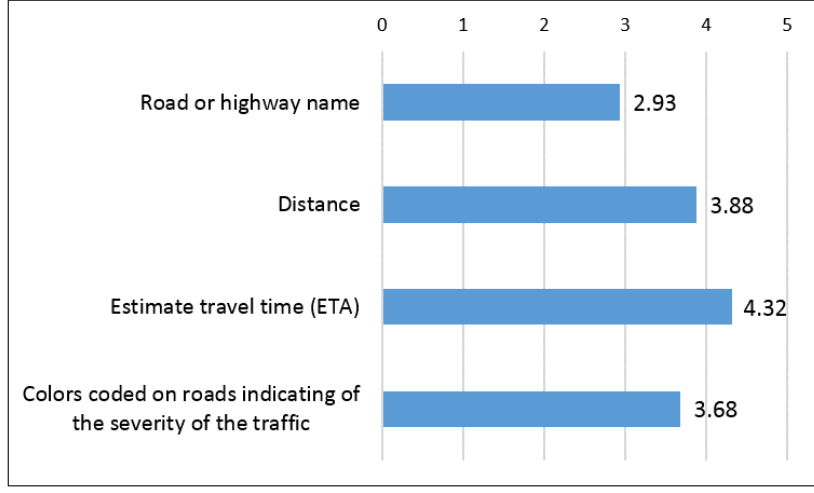

(b) Q12. Criteria rate. (w/o traffic image)

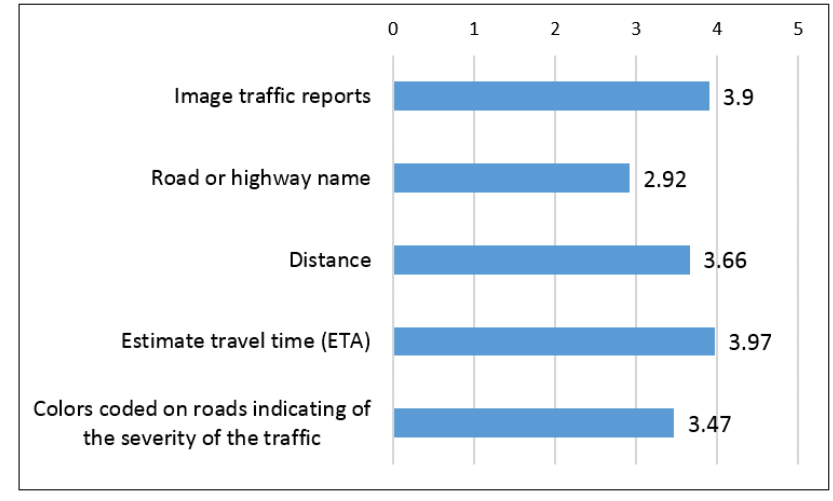

(c) Q14. Criteria rate. (w/ image)

Fig. 6. Survey Questionnaire Results (Q11 - Q14)

Route Choice Behavior. The purpose of Q11 to Q14 was to observe the route choice behavior in a real scenario when users selected a route by using current, traditional route selection criteria versus when users were provided with real traffic images. When users were asked to select either route 1 or route 2 by using traditional methods (without traffic images), $79 \%$ selected route 1 , and $21 \%$ selected route 2 . Then, participants rated the criteria their decision was based on, and ETA was ranked at 4.32 , followed by distance at 3.88. Afterwards, participants were shown the same map and routes along with real traffic images and were asked to select a route. This time, $49 \%$ selected route 1 and $51 \%$ selected route 2 . There was a preference change, with a $30 \%$ decrease in selection of route 1 and a 30\% increase in selection of route 2 after viewing images. After viewing traffic images and selecting a route, participants were again asked to rate what their decision was based on. The rank for using traffic images was 3.9, and ETA was 3.97 (a 0.35 decrease compared to not using traffic images). Details are shown in Figure 6.

Influence of Traffic Images. For Q15, the participants rated how supportive and important traffic images were, with $25 \%$ rating them essential and 58\% rating them important, as shown in Figure 7(b). Also, to recognize why users would look at realtime traffic images, participants were asked to rate nine items (Q16). The top three items were "To see traffic conditions for myself"; "To get an extra level of detail"; and "To confirm the traffic condition when there is doubt about the reliability of other criteria such as ETA," as shown in Figure 7(c). Finally, participants were asked what influence real-time traffic images would have; $71 \%$ responded that images helped to determine route choice, and $62 \%$ responded that images prepared them for traffic conditions ahead, as seen in Figure 7(d).

\section{DISCUSSION AND FUTURE WORK}

Several issues require further research. Building an optimized user interface to enable drivers to interact with the navigation system while driving is essential. Issues such as passenger safety and reducing cognitive load must be further examined through an analytical user study.

Selecting the most relevant tweets to be included in the tweet digest is a non-trivial task when the number of tweets is large and to effectively capture the semantic meaning of events on the route. Other approaches can be explored to improve tweet selection by using other criteria, such as user reputation, or by crowdsourcing this task to people willing to help in real time. Driver feedback on tweets can also help to eliminate improper or malicious tweets.

The overall architecture of SVN should be designed as a scalable online service for all commuters to share traffic information. The architecture should accommodate a large amount of users uploading or downloading data at the same time. A distributed architecture, or for example, cloudlets [18], can provide a natural solution to provide scalability. Moreover, we favor a distributed architecture due to the fact that most commutes occur between home and work within a reasonably stable and small geographic region. A local server cluster or cloudlet can handle the majority of requests in its locality. 


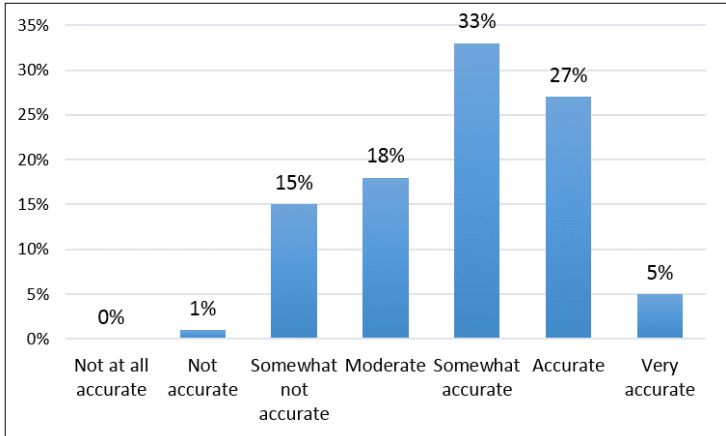

(a) Q9. Accuracy of ETA

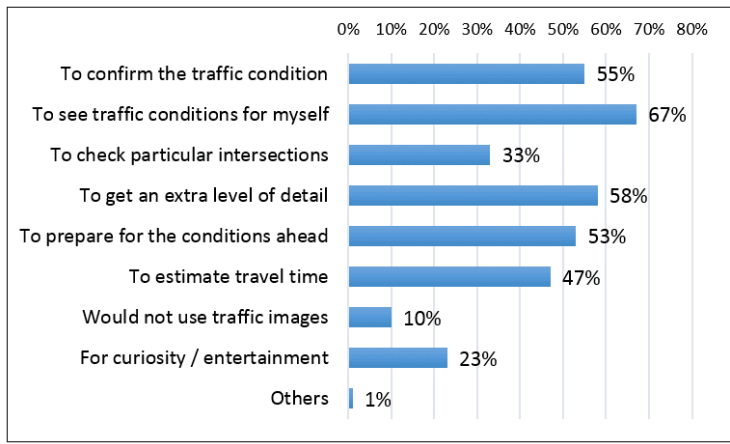

(c) Q16. Uses of traffic images (Multiple answer question)

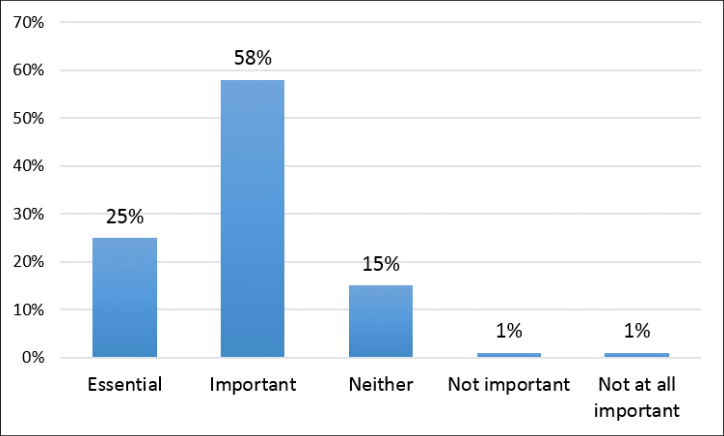

(b) Q15. Rate how image would support route choice

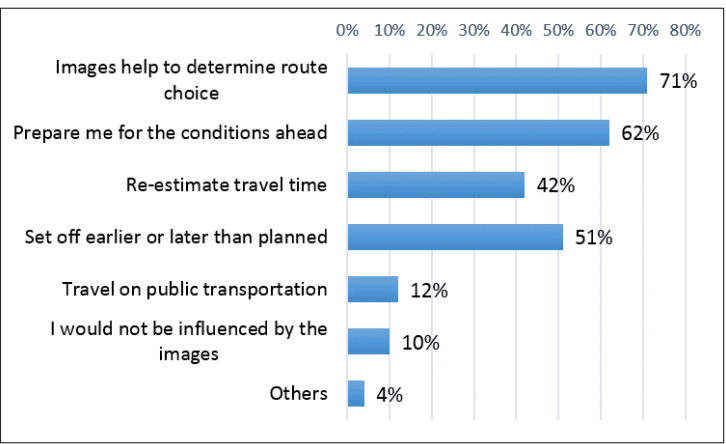

(d) Q17. Influence in traffic images (Multiple answer question)

Fig. 7. Survey Questionnaire Results (Q9, Q15, Q16, Q17)

\section{CONCLUSION}

This paper proposes a social vehicular navigation system where driver-provided geo-tagged traffic image reports can assist other drivers in their route selection. The traffic reports are called NaviTweets, and their summaries called Traffic Digests are periodically produced and sent to drivers. The paper presents the system design for the SVN prototype implemented on an Android platform, along with the results from a questionnaire survey to evaluate the usage of traffic images in route choice.

\section{REFERENCES}

[1] Waze. http://www.waze.com

[2] Inrix Traffic. http://www.inrixtraffic.com

[3] Trapster. htts://www.trapster.com

[4] New Cities Foundation (2012), "Connected Commuting: Research and analysis on the New Cities Foundation Task Force in San Jose," http://www.newcitiesfoundation.org/wp-content/uploads/New-CitiesFoundation-Connected-Commuting-Full-Report.pdf.

[5] S. Smaldone, L. Han, P. Shankar, and L. Iftode, "RoadSpeak: Enabling voice chat on roadways using Vehicular Social Networks," in Proc. of the 1st Workshop on Social Network Systems (Glasgow, Scotland, April 0104, 2008), SocialNets'08.

[6] W. Sha, D. Kwak, B. Nath, and L. Iftode, "Social Vehicle Navigation: Integrating shared driving experience into vehicle navigation," in Proc. of the 14th Workshop on Mobile Computing Systems and Applications (Jekyll Island, Feb. 26-27, 2013), HotMobile '13.

[7] M. Gerla and L. Kleinrock, "Vehicular networks and the future of the mobile internet," Computer Networks, 55 (2), Feb. 2011, 457-469.

[8] AM. Tawfik, H.A Rakha, and S.D. Miller, "Driver route choice behavior: Experiences, perceptions, and choices," in Proc. Intelligent Vehicles Symposium (IV), 2010 IEEE, vol., no., pp.1195,1200, 21-24 June 2010.

[9] G.M. Ramos, E. Frejinger, W. Daamen, and S. Hoogendoorn, “A revealed preference study on route choices in a congested network with real-time information," in Proc. 13th International Conference on Travel Behaviour Research Toronto 15-20, July 2012.

[10] R. Selten, T. Chmura, T. Pitz, S. Kube, and M. Schreckenberg, "Commuters route choice behaviour," Games and Economic Behavior, Vol. 58, pp: 394-406, Feb. 2007.

[11] B. L. Hanchett, "Traffic condition information system," United States Patent: 5,396,429. Mar. 7, 1995.

[12] T.B.W. Adam, I.M. Atkinson, and M.J. Dixon, "Navigation device displaying traffic information," United States Patent: US2007/0118281A1. May 24, 2007.

[13] S. Speirs and P. Whitehead, "Impact of Images from Traffic Cameras on Journey Planning," in Proc. Transport Information and Control - RTIC 2008 and ITS United Kingdom Members' Conference, IET, vol., no., pp. 1,8, 20-22 May 2008.

[14] D.J. Parkyns and M. Bozzo, "CCTV Camera sharing for improved traffic monitoring," in Proc. Road Transport Information and Control - RTIC 2008 and ITS United Kingdom Members' Conference, IET, May 2008.

[15] 511 org websites, NJ: www.511nj.org/, NY: 511ny.org/, VA: http://www.511virginia.org/, MN: http://www.511mn.org/ SF: 511.org/.

[16] L. Higgins, A. Nelson, T. Geiselbrecht, and B. Ullman, "Understanding the decision-making process for drivers faced with lane restriction or closures on Wisconsin highways," Technical Report, Texas A\&M Transportation Institute, February 2013.

[17] R. D. Golledge, "Defining the Criteria used in Path Selection," Ettema, D.F., Timmermans, H.J.P. (Eds.), Activity-Based Approaches to Travel Analysis. Pergamon, Oxford, pp. 151-170, 1997.

[18] M. Satyanarayanan, P. Bahl, R. Caceres, and N. Davies, "The Case for VM-Based Cloudlets in Mobile Computing," Pervasive Computing, IEEE, vol.8, no.4, pp.14,23, Oct.-Dec. 2009.

[19] Qualtrics. http://www.qualtrics.com/

[20] Google maps. https://developers.google.com/maps/

[21] OsmAnd (OpenStreetMap Automated Navigation Directions). http://osmand.net/

[22] Instragram. http://instagram.com/ 